\section{What's In This Issue?}

Letter from the founding facilitator for issue 5

It is a pleasure to publish Volume 1, Issue 5 of the RRNMF Neuromuscular Journal. Once again we have many interesting articles in our new issue and some are in categories that are a bit different from our first 4 issues. In addition to this brief introduction, we have three other pieces in the "What's on Your Mind?" Category. The first is another outstanding article by my good friend Dr. Josh Freeman on the topic of how the Covid-19 pandemic disproportionately strikes the most vulnerable members of our society and how this in part can be attributed to longstanding health care disparities on our system. As I said in the last issue, Josh has had a blog site for years and I thought we should allow him to publish his some of his great pieces in our new journal. The next editorial is by Dr. Raghav Govindarajan, my neuromuscular partner at the University of Missouri. Dr. G has written a wonderful piece about the millennial generation and how as they come to age they will have an impact on everything, including our profession of neurology. The final piece in this section is something really different for a neurology journal. It is my list and accompanying explanation of Rick Barohn's Ranking List of the North American Art Museums. Art is one of my hobbies and over the years I have had the immense privilege of traveling around the world to attend medical and neurology meetings. I always made it a point to visit the local art museum and I developed an "NIH style" ranking system for the ones in the USA and Canada. I won't say any more in these comments and you can learn more about how the list evolved in the article. When I launched this journal I made a point that we welcomed articles outside of the world of neuromuscular disease, neurology, and medicine, so here is the first one. Hopefully we will receive more.

In the original neuromuscular section, beginning with the "New Stuff" we have a terrific article by the Cleveland Clinic neuromuscular group authored by Drs. Tiffany PikeLee and Yuebing Li describing three cases of CIDP that also had another underlying autoimmune disorder. I recall we had a number of cases in our 1989 CIDP paper in which a variety of other diseases were associated including Hodgkin's, inflammatory bowel disease, and chronic hepatitis, and we introduced the concept of "concurrent diseases" with CIDP (Barohn RJ et. al Archives of Neurology 1989; 46:878884). Here the authors have a case of primary sclerosing cholangitis (not previously reported with CIDP), a case of ITP, and a case with a very high ANA. We then have four interesting single case reports: Sjögren's syndrome and a sensory motor autonomic neuropathy (Farheen et al); GBS after a Shingrix vaccine (Zafar et al); motor neuron disease in an HIV patient who stopped taking antiretroviral therapy (de Bruin et al); and a CIDP case with IgG tubular antibodies that was difficult to treat (Giacobbe et al). These are all welcome contributions to the neuromuscular literature. In the Visual Stuff category, Dr. Govindarajan describes how ptosis improved rapidly after initiation of plasmapheresis and he makes the point that this may be the first objective sign of improvement after the initiation of plasmapheresis. In looking at his figures I noted in addition to upper lid ptosis there is what I call lower lid reverse ptosis where the lower lid sags downward. This also improved with the plasmapheresis.

Finally, I am reprinting a paper I published in 1997 in a rather obscure annual neurology review publication from India called Advances in Clinical Neurosciences. Since I doubt anyone outside of India has seen it, and I always liked this paper, I asked the editors and publishers of the original annual if I could republish the paper in the RRNMF Neuromuscular Journal and they gave me permission to do so. It is a project I did as a neurology resident in which I collected a dozen cases of what we called then reflex sympathetic dystrophy (now referred to as complex regional pain syndrome) and all of the cases had an underlying peripheral neuropathy. In addition, all had abnormal radionuclide three-phase bone scans.

Recently two University of Missouri medical students began assisting in the editorial process of this journal. Jiji Oufattole is currently an MS2, and Breanna Tuhlei is an MD/ $\mathrm{PhD}$ student (in neuroimmunology!) and she is in the MS3 year of her training. They have been assisting in copy editing and as we move forward they will be Co-Managing Editors of the journal. Thank you Jiji and Breanna! I still get an enormous amount of help and support from Marianne Reed and Pam LeRow in the digital publishing unit at KU. I could not get these issues out without them. Thank you Marianne and Pam! I also am thankful for the constant assistance of Amanda Sebok who is the senior executive administrative assistant in the EVC of Health Affairs office at the University of Missouri. I could not function professionally without her.

I believe the RRNMF Neuromuscular Journal readership will enjoy these publications. Please continue to send in articles for upcoming issues. Be thankful every day. I know I am. Have a happy minimalistic Thanksgiving and please be safe and wear your masks!

Rick 